\title{
Date of New Tumor Event
}

National Cancer Institute

\section{Source}

National Cancer Institute. Date of New Tumor Event. NCI Thesaurus. Code C156855.

The date of the new tumor event. 